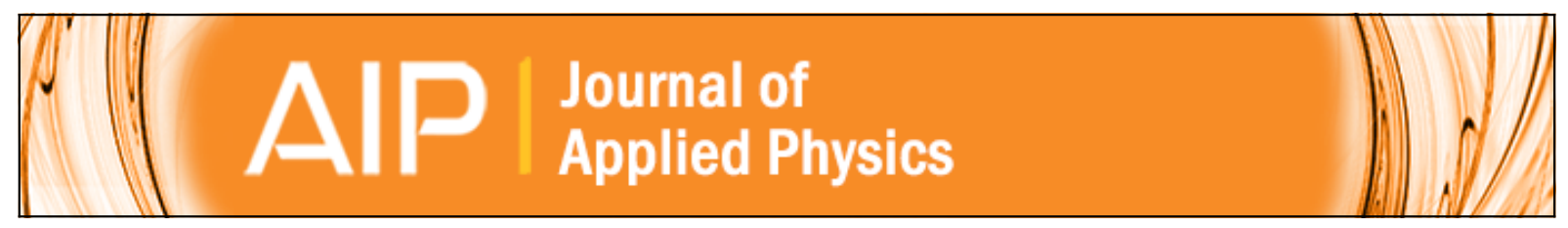

\title{
Nonlinear optical figures of merit of processible composite of poly(2-methoxy,5-(2'- (ethyl)hexyloxy)-p-phenylene vinylene) and poly(methyl methacrylate)
}

\author{
Y. Lin, J. Zhang, L. Brzozowski, E. H. Sargent, and E. Kumacheva
}

Citation: Journal of Applied Physics 91, 522 (2002); doi: 10.1063/1.1420760

View online: http://dx.doi.org/10.1063/1.1420760

View Table of Contents: http://scitation.aip.org/content/aip/journal/jap/91/1?ver=pdfcov

Published by the AIP Publishing

\section{Articles you may be interested in}

Connection between the conformation and emission properties of poly[2-methoxy-5-(2'-ethyl-hexyloxy)-1,4phenylene vinylene] single molecules during thermal annealing

Appl. Phys. Lett. 106, 123304 (2015); 10.1063/1.4916611

Enhancement of optical gain and amplified spontaneous emission due to waveguide geometry in the conjugated polymer poly[2-methoxy-5-(2'-ethylhexyloxy)-p-phenylene vinylene]

Appl. Phys. Lett. 102, 073303 (2013); 10.1063/1.4793422

A planar organic near infrared light detector based on bulk heterojunction of a heteroquaterphenoquinone and poly[2-methoxy-5-( 2 ' -ethyl-hexyloxy)-1, 4-phenylene vinylene]

J. Appl. Phys. 104, 114508 (2008); 10.1063/1.3033376

Optical emission from nano-poly[2-methoxy-5-( 2 ' -ethyl-hexyloxy)- $p$-phenylene vinylene] arrays J. Appl. Phys. 98, 074304 (2005); 10.1063/1.2076446

Optical studies of electric fields in poly(2-methoxy-5-ethyl ( 2 ' -hexyloxy) para-phenylene vinylene) light-emitting diodes

Appl. Phys. Lett. 74, 3714 (1999); 10.1063/1.123238

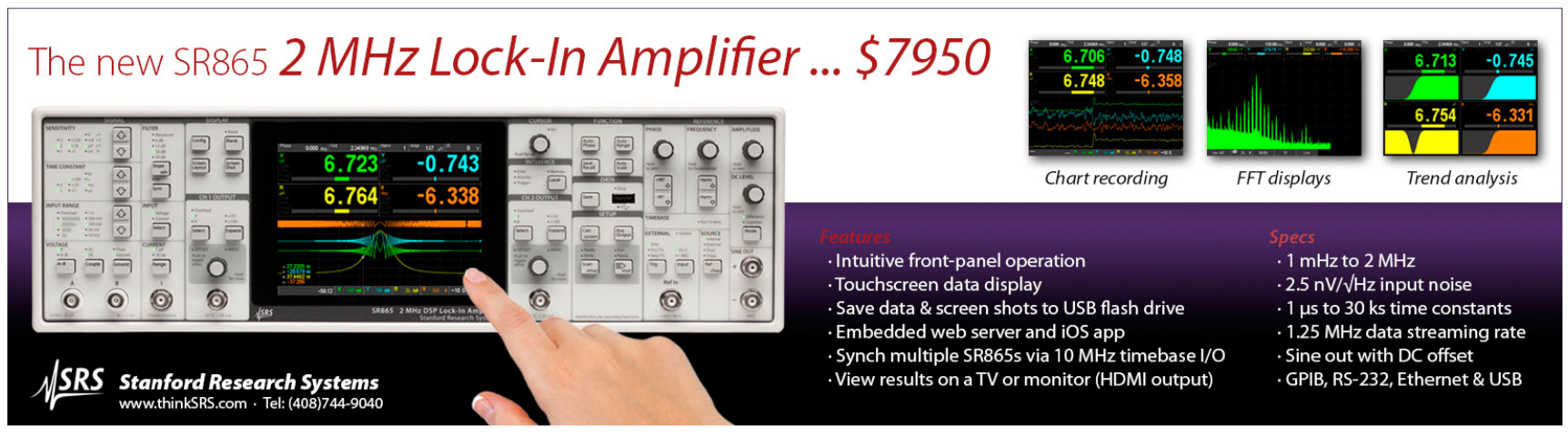




\title{
Nonlinear optical figures of merit of processible composite of poly(2-methoxy,5-(2'-(ethyl)hexyloxy)-p-phenylene vinylene) and poly(methyl methacrylate)
}

\author{
Y. Lin ${ }^{a}$ \\ Edward S. Rogers Sr. Department of Electrical and Computer Engineering, University of Toronto, \\ Ontario M5S 3G4, Canada \\ J. Zhang \\ Department of Chemistry, University of Toronto, Ontario M5S 3H6, Canada \\ L. Brzozowski and E. H. Sargent \\ Edward S. Rogers Sr. Department of Electrical and Computer Engineering, University of Toronto, \\ Ontario M5S 3G4, Canada \\ E. Kumacheva \\ Department of Chemistry, University of Toronto, Ontario M5S 3H6, Canada
}

(Received 30 May 2001; accepted for publication 29 September 2001)

\begin{abstract}
We report ultrafast nonlinear optical figures of merit for a highly processible guest-host blend of poly(2-methoxy,5-(2'-(ethyl)hexyloxy)-p-phenylene vinylene) with poly(methyl methacrylate). Our experiments employ $120 \mathrm{fs}$ pulses at $840 \mathrm{~nm}$ and are designed to eliminate slow thermal nonlinearity and focus exclusively on ultrafast electronic nonlinearity. We report a two-photon absorption coefficient $\beta$ of $1.5 \pm 0.2 \mathrm{~cm} / \mathrm{GW}$, a nonlinear refraction coefficient $n_{2}$ of $-(2.1 \pm 0.2) \times 10^{-13}$ $\mathrm{cm}^{2} / \mathrm{W}$ and a two-photon figure of merit $T$ of 0.6 . The blend hybridizes the desirable features of nonlinearity and processibility of its two constituents to provide (1) ultrafast response one to two orders of magnitude faster than achievable in electronic switching devices; (2) a two-photon figure of merit compatible with harnessing this nonlinearity in a practical optical device geometry; and (3) a materials system and processing methodology compatible with spin-coating and photopatterning in an ambient environment. (C) 2002 American Institute of Physics. [DOI: 10.1063/1.1420760]
\end{abstract}

Nonlinear optical materials provide a basis for optical switching, modulation, and limiting. Since photons are essentially noninteracting in vасиo, nonlinear materials are needed for one optical signal to influence another, providing a generalized basis for all-optical signal processing. If femtosecond ultrafast nonlinearities can be harnessed, the resulting device speeds may provide orders of magnitude improvement over what can be achieved even prospectively in the electronic domain.

Nonresonant third-order nonlinearity, wherein refractive index depends near-instantaneously on the local intensity of light, has attracted significant attention in this regard, including in $\pi$-conjugated polymers. ${ }^{1-13}$ Such materials may exhibit promising nonresonant nonlinear coefficients and may in certain cases be highly processible. They may be deployed in organic or inorganic guest-host systems. As such they offer the opportunity to combine desirable properties processibility, speed and strength of response, extent of absorption, photopatternability, and spectral features to achieve a material designed and deployed to meet the specifications of a particular application.

Poly(p-phenylene vinylene) (PPV) and its derivatives have received particular attention across the field of photonics as a result of demonstrations of both efficient electrolu-

${ }^{\text {a)} E l e c t r o n i c ~ m a i l: ~ y u a n k u n . l i n @ u t o r o n t o . c a ~}$ minescence and an appreciable ultrafast optical nonlinearity. ${ }^{1-6,8-11,13-15}$ However, PPV is insoluble. Thin films are thus typically prepared by thermal conversion of a soluble nonconjugated intermediate, or precursor, polymer. This presents a major challenge in deploying PPV in such a way to obtain optical quality, highly transparent films required for optical waveguiding. PPV semicrystalline regions formed during conversion of the precursor polymer, for example, result in appreciable scattering of light out of an intended single mode. ${ }^{4,5}$

One prospective way to combine the promising nonlinear properties of PPV with suitable processibility and linear properties is to prepare composites of PPV within suitablychosen host materials. ${ }^{3-5,10,11,16}$ We report herein results of our investigations into the guest-host polymer blend poly(2-methoxy,5-(2'-(ethyl)hexyloxy)-p-phenylene vinylene) (MEH-PPV) with a poly(methyl methacrylate) (PMMA) host. We selected as dopant MEH-PPV since it is a soluble derivative of PPV with lower intrinsic absorption than PPV. ${ }^{6}$ PMMA is a good candidate for the host material since it is amorphous and has good optical transparency and is readily processible into films. In the present work, we focus on an MEH-PPV/PMMA polymer blend containing 0.2 wt $\%$ MEH-PPV. For the composite material we obtain a nonlinear Kerr coefficient $n_{2}=-(2.1 \pm 0.2) \times 10^{-13} \mathrm{~cm}^{2} / \mathrm{W}$, nonlinear absorption coefficient $\beta=1.5 \pm 0.2 \mathrm{~cm} / \mathrm{GW}$, and two-photon figures of merit 0.6 for ultrafast electronic non- 


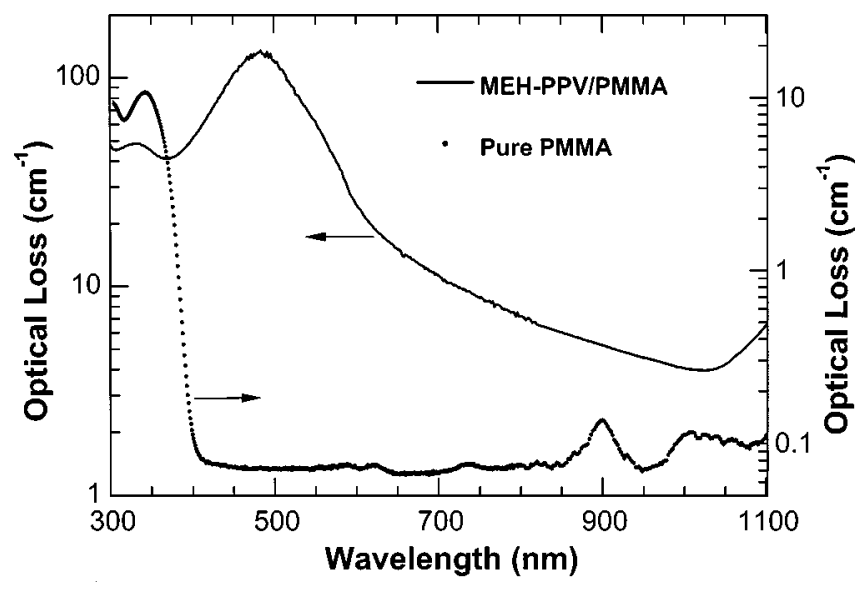

FIG. 1. Linear absorption spectra of pure PMMA (dotted) and MEH-PPV/ PMMA composite (solid line).

linearity probed using $120 \mathrm{fs}$ pulses centered at $840 \mathrm{~nm}$.

PMMA and MEH-PPV were separately dissolved in pyridine. These two solutions were then combined and stirred for $20 \mathrm{~min}$. The homogeneous pink solution was evaporated to remove most of the solvent and the concentrated solution was then poured into a mold with a Teflon ${ }^{\mathrm{TM}}$ substrate. The sample was dried at room temperature overnight and subsequently in an oven at $70^{\circ} \mathrm{C}$ for $10 \mathrm{~h}$ to remove the pyridine. The sample was then passed into films at $140^{\circ} \mathrm{C}$ and slowly cooled to room temperature to avoid the shrinkage of the films. The weight ratio of MEH-PPV to PMMA was $0.2 \%$ throughout the present work. The thickness of the composite film was $0.2 \mathrm{~mm}$.

The MEH-PPV/PMMA composites were characterized for linear optical loss using a Cary 500 UV-VIS-NIR spectrophotometer. The nonlinear properties of the films were measured using amplified pulses from a Coherent MIRA 900 femtosecond Ti:Sapphire laser at $840 \mathrm{~nm}$. Autocorrelation results indicated a pulse width of $120 \mathrm{fs}$. In order to ensure that fast electronic nonlinear optical effects were observed rather than slow thermo-effects, a MEDOX Pockels cell was used to provide an output pulse repetition rate of $5 \mathrm{kHz}$. The laser beam was focused onto the sample using a lens of focal length $f=100 \mathrm{~mm}$. The beam profiles at the sample surface were measured to have $1 / e^{2}$ diameter of 39-49 $\mu \mathrm{m}$. An aperture with linear beam transmittance $S=0.1$ was used for all closed-aperture measurements.

The linear optical losses, including both absorption and scattering, are shown in Fig. 1 for both MEH-PPV/PMMA composites and pure PMMA. For the composite, a peak is evident near $485 \mathrm{~nm}$ with peak absorption value $135 \mathrm{~cm}^{-1}$. Above $600 \mathrm{~nm}$, the optical loss gradually decreases to a minimum near $1050 \mathrm{~nm}$. For the purposes of the present work, the pertinent wavelength for loss measurements is 840 $\mathrm{nm}$, the wavelength at which nonlinear experiments were carried out and linear loss was found to be $6.2 \mathrm{~cm}^{-1}$. In contrast, pure PMMA is essentially transparent from 380 to $1100 \mathrm{~nm}$. A small peak around $900 \mathrm{~nm}$ results from $\mathrm{C}-\mathrm{H}$ overtones. ${ }^{17}$ The absorption spectrum of MEH-PPV/PMMA exhibits similar features to those of pure MEH-PPV. ${ }^{6,18}$

Figure 2 shows the result of a single-beam open-aperture

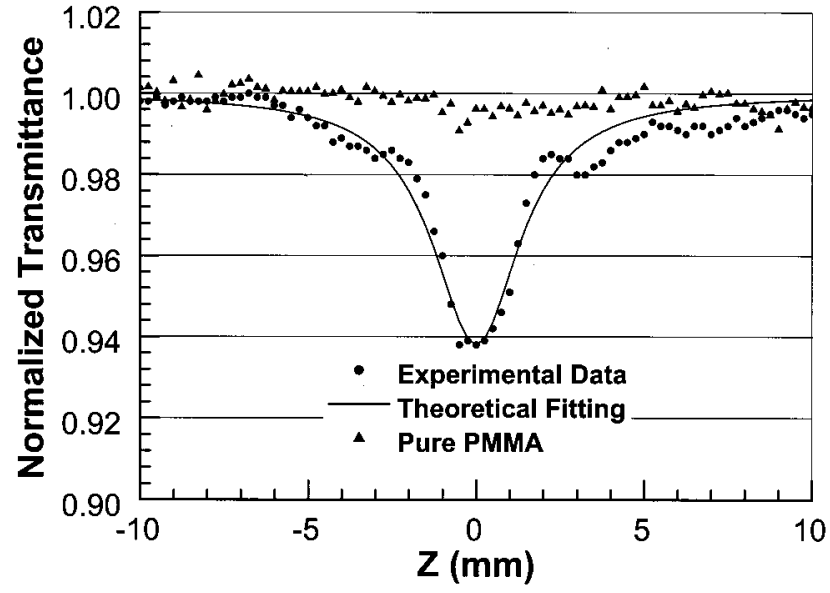

FIG. 2. Normalized open-aperture Z-scan transmittances of MEH-PPV/ PMMA (circles) and pure PMMA (triangles) film samples using $120 \mathrm{fs}$ pulses at $\lambda=840 \mathrm{~nm}$. The solid line is a theoretical fitting.

$Z$-scan measurement at $840 \mathrm{~nm}$ for MEH-PPV/PMMA composites and pure PMMA. The laser intensity at the focal point is calculated to be $6.12 \mathrm{GW} / \mathrm{cm}^{2}$. The calculation is made using the expressions of Sheikh-Bahae et al. ${ }^{19}$ Multiphoton absorption from pure PMMA is not observed in Fig. 2. As seen in Fig. 1, PMMA is transparent down to $380 \mathrm{~nm}$, so only weak three-photon absorption could contribute to nonlinear processes at $840 \mathrm{~nm}$. The normalized transmittance from MEH-PPV/PMMA composites exhibits an almost symmetric valley with respect to the focus $(Z=0)$, which is the character of the nonlinear absorption from multiphoton absorption. ${ }^{19}$

For the pure MEH-PPV material, thee is an absorption peak around $500 \mathrm{~nm}$. The observed nonlinear absorption is thus from the two-photon absorption due to MEH-PPV and not from PMMA. For a small third-order nonlinear loss and for a Gaussian temporal shape pulse, the normalized transmittance can be described by ${ }^{20}$

$$
T(Z)=1-\frac{\beta I_{0} L_{\text {eff }}}{2 \sqrt{2}} \frac{1}{1+Z^{2} / Z_{0}^{2}},
$$

where $\beta$ is the two-photon absorption coefficient, $L_{\text {eff }}=(1$ $\left.-e^{-\alpha L}\right) / \alpha$, with $L$ the sample thickness, $\alpha$ the linear absorption coefficient, and $Z_{0}=\pi u_{0}^{2} / \lambda$ with $u_{0}$ the beam radius at focal point. At $840 \mathrm{~nm}, L_{\text {eff }}=0.19 \mathrm{~mm}$. A theoretical fitting to the experimental data is shown in Fig. 2. From the fitting we obtain $\beta=1.5 \pm 0.2 \mathrm{~cm} / \mathrm{GW}$ and $w_{0}=21 \mu \mathrm{m} . w_{0}$ is in agreement with beam profiler measurements.

The nonlinear refractive index $n_{2}$ was obtained from closed-aperture Z-scan measurements. To obtain the refractive nonlinearity in the presence of two-photon absorption, the closed-aperture scan was first divided by the openaperture scan. The result is shown in Fig. 3. The experimental data were fitted to the expression given by Sheik-Bahae et al., ${ }^{19}$ which relates the normalized transmittance $T\left(Z, \Delta \phi_{0}\right)$ directly to phase change $\Delta \phi_{0}$, 


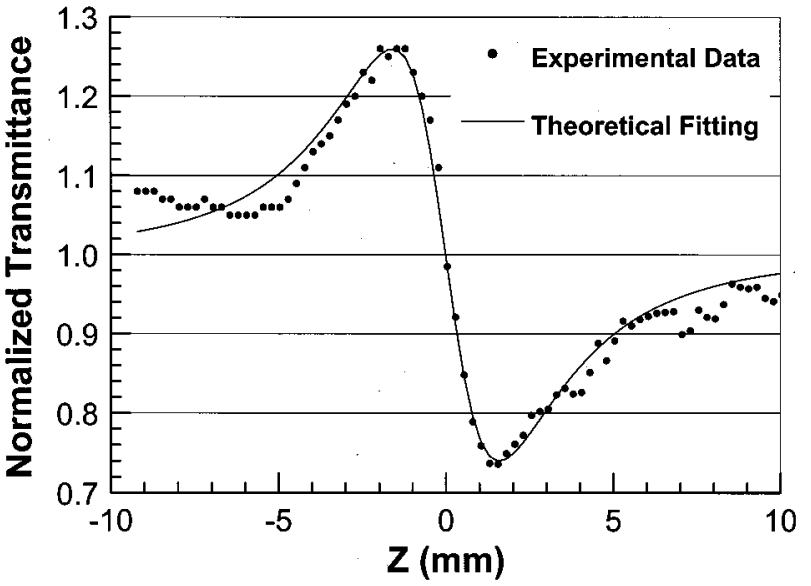

FIG. 3. Normalized closed-aperture $(S=0.1) Z$-scan transmittance of 0.2 $\mathrm{mm}$ thick MEH-PPV/PMMA film sample using femtosecond pulses at $\lambda$ $=840 \mathrm{~nm}$ with $I_{0}=6 \mathrm{GW} / \mathrm{cm}^{2}$. The open-aperture scan was subtracted. The solid line is a theoretical fitting.

$$
T\left(Z, \Delta \phi_{0}\right) \approx 1+\frac{4 \Delta \phi_{0} x}{\left(1+x^{2}\right)\left(9+x^{2}\right)},
$$

where $x=Z / Z_{0}$. Nonlinear least-squares fitting with $\Delta \phi_{0}$ and $Z_{0}$ as parameters yielded $\Delta \phi_{0}=-1.28$ and $Z_{0}$ $=0.00184$. From the relation $\Delta \phi_{0}=2 \pi \Delta n L_{\text {eff }} / \lambda$, we obtain refractive index change at the focal point of $\Delta n=-9.02$ $\times 10^{-4}$ and Kerr coefficient $n_{2}=-(2.1 \pm 0.2) \times 10^{-13}$ $\mathrm{cm}^{2} / \mathrm{W}$. From $Z_{0}$, the beam size at the focus point is calculated to be $44 \mu \mathrm{m}$ in good agreement with the beam profiler measurement. More rigorous calculation including the correction from the aperture size $S=0.1$ gives the same $n_{2}$ value to within $5 \%$ uncertainty. ${ }^{19}$ The negative sign in $n_{2}$ indicates that the material has a defocusing nonlinearity. This is consistent with the fact that the laser wavelength sits between the one- and two-photon absorption edges of MEH-PPV.

For a third-order nonlinear material to provide a useful phase change before it is substantially absorbed, it is essential that the two-photon figure of merit $T=\beta \lambda / n_{2}$ be less than 1 . The results reported above indicate that the $0.2 \%$ MEH-PPV/PMMA composite has figure of merit of 0.6 at $840 \mathrm{~nm}$ for 120 fs pulses.

The one-photon figure of merit, $W$, is defined as $W$ $=\Delta n / \alpha \lambda$, where $\alpha$ is the absorption coefficient. For $\Delta n$ $=9.02 \times 10^{-4}, \alpha=6.2 \mathrm{~cm}^{-1}$, and $\lambda=840 \mathrm{~nm}$, the figure of merit $W=1.7$. The value of $W$ is promising but marginal. We note that the absorption measured includes all sources of loss, both intrinsic and extrinsic, and that it may be possible to reduce extrinsic loss (e.g., scattering due to sample inhomogeneities). Thus, unlike the more fundamental figure of merit $T$, which for the material presented herein is good for nonlinear device performance, the figure of merit $W$ has a substantial technological component susceptible to further optimization.

It is worthwhile to compare these results with those obtained for pure MEH-PPV and PPV materials. Samoc et al. ${ }^{1}$ have obtained $n_{2} \geqslant 10^{-11} \mathrm{~cm}^{2} / \mathrm{W}$ and a nonlinear absorption coefficient $\beta$ of $80 \mathrm{~cm} / \mathrm{GW}$ at $800 \mathrm{~nm}$, yielding $T \leqslant 0.6$, measured by degenerate four-wave mixing for the pure PPV materials (sign of $n_{2}$ has not been determined). The same group $^{2}$ also reported $Z$-scan measurements at $800 \mathrm{~nm}$ on MEH-PPV in solution. The value of $n_{2}$ has been determined from the slope of the $n_{2}$-versus-concentration dependence, as the authors have mentioned, which in principle is substantially different from the value of $n_{2}$ for the pure material in the solid phase. They obtained $n_{2}=-2.1 \times 10^{-12} \mathrm{~cm}^{2} / \mathrm{W}, T$ $=4.7$ for $\mathrm{MEH}-\mathrm{PPV}$ measured in THF and $n_{2}=-3.4$ $\times 10^{-12} \mathrm{~cm}^{2} / \mathrm{W}, T=4.2$ for MEH-PPV measured in $\mathrm{CHCl}_{3}$.

In summary, a guest-host MEH-PPV/PMMA polymer blend was synthesized and its linear and nonlinear optical properties were investigated with a goal of controlling its optical properties for a practical application. Third-order nonlinear optical properties were measured by the singlebeam $Z$-scan technique at $840 \mathrm{~nm}$ using $120 \mathrm{fs}$ pulses in the ultrafast electronic nonlinear regime.

The authors thank Michael O. Wolf in the University of British Columbia for providing us with MEH-PPV samples and Peter W. Smith in the University of Toronto for laboratory use. One of the authors (Y.L.) thanks NSERC for a postdoctoral fellowship.

${ }^{1}$ A. Samoc, M. Samoc, M. Woodruff, and B. Luther-Davies, Opt. Lett. 20, 1241 (1995)

${ }^{2}$ M. Samoc, A. Samoc, B. Luther-Davies, Z. Bao, L. Yu, B. Hsieh, and U. Scherf, J. Opt. Soc. Am. B 15, 817 (1998).

${ }^{3}$ M. Woodruff, M. Samoc, and B. Luther-Davies, Chem. Mater. 8, 2586 (1996).

${ }^{4}$ A. Samoc, M. Samoc, and B. Luther-Davies, Proc. SPIE 3147, 166 (1997).

${ }^{5}$ D. L. Wise, G. E. Wnek, D. J. Trantolo, T. M. Cooper, and J. D. Gresser, Photonic Polymer Systems (Marcel Dekker, New York, 1998), p. 373.

${ }^{6}$ S. J. Martin, D. D. C. Bradley, and H. Mellor, Phys. Rev. B 59, 15133 (1999).

${ }^{7}$ D. Guo, S. Mazumdar, S. N. Dixit, F. Kajzar, F. Jarka, Y. Kawabe, and N. Peyghambarian, Phys. Rev. B 48, 1433 (1993).

${ }^{8}$ A. Mathy, K. Ueberhofen, R. Schenk, H. Gregorius, R. Garay, K. Müllen, and C. Bubeck, Phys. Rev. B 53, 4367 (1996).

${ }^{9}$ J. Swiatkiewicz, P. N. Prasad, and F. E. Karasz, J. Appl. Phys. 74, 525 (1993).

${ }^{10}$ Th. Gabler, R. Waldhäusl, A. Bräuer, F. Michelotti, H. H. Hörhold, and U. Bartuch, Appl. Phys. Lett. 70, 928 (1997).

${ }^{11}$ C. J. Wung, Y. Pang, P. N. Prasad, and F. E. Karasz, Polymer 32, 605 (1991).

${ }^{12}$ S. R. Marder, B. Kippelen, A. K. Jen, and N. Peyghambarian, Nature (London) 388, 845 (1997).

${ }^{13}$ R. Rangel-Rojo, S. Yamada, H. Matsuda, and D. Yankelevich, Appl. Phys. Lett. 72, 1021 (1998)

${ }^{14}$ R. H. Friend et al., Nature (London) 397, 121 (1999).

${ }^{15}$ F. Hide, M. A. Díaz-García, B. J. Schwartz, M. R. Anderson, Q. Pei, and A. J. Heeger, Science 273, 1833 (1996).

${ }^{16}$ R. W. Boyd, R. J. Gehr, G. L. Fischer, and J. E. Sipe, Pure Appl. Opt. 5, 505 (1996).

${ }^{17}$ C. H. Seager, M. Sinclair, D. McBranch, A. J. Heeger, and G. L. Baker, Synth. Met. 49, 91 (1992).

${ }^{18}$ N. C. Greenham, X. Peng, and A. P. Alivisatos, Phys. Rev. B 54, 17628 (1996).

${ }^{19}$ M. Sheik-Bahae, A. A. Said, T. Wei, D. J. Hagan, and E. W. Van Stryland, IEEE J. Quantum Electron. 26, 760 (1990).

${ }^{20}$ M. G. Kuzyk and C. W. Dirk, Characterization Techniques and Tabulations for Organic Nonlinear Optical Materials (Marcel Dekker, New York, 1998), p. 655 